\title{
Elaboração da versão em português do Psychotherapy Process Q-Set
}

\author{
Development of a Portuguese version of the Psychotherapy Process Q-Set
}

\author{
Fernanda Barcellos Serralta ${ }^{1}$, Maria Lúcia Tiellet Nunes², Cláudio Laks Eizirik ${ }^{3}$ \\ 1 Psicóloga. Mestre em Psicologia Clínica, Pontifícia Universidade Católica do Rio Grande do Sul (PUCRS), Porto Alegre, RS. Doutoranda em \\ Psiquiatria, Universidade Federal do Rio Grande do Sul (UFRGS), Porto Alegre, RS. Professora adjunta, Curso de Psicologia, Universidade \\ Luterana do Brasil (ULBRA), Canoas, RS. ${ }^{2}$ Psicóloga. Doutora em Psicologia, Universidade Livre de Berlim, Berlim, Alemanha. Professora titular, \\ Faculdade de Psicologia, PUCRS, Porto Alegre, RS. Coordenadora, Programa de Pós-Graduação em Psicologia, Faculdade de Psicologia, PUCRS, \\ Porto Alegre, RS. ${ }^{3}$ Médico psiquiatra. Doutor em Medicina, UFRGS, Porto Alegre, RS. Professor adjunto, Departamento de Psiquiatria e Medicina \\ Legal, UFRGS, Porto Alegre, RS. Analista didata, Sociedade Psicanalítica de Porto Alegre, Porto Alegre, RS. Presidente da International \\ Psychoanalytic Association (IPA).
}

\section{Resumo}

Introdução: No Brasil, a pesquisa em psicoterapia encontra-se em desenvolvimento inicial; ainda não há estudos sistemáticos do processo terapêutico, e poucas são as medidas disponíveis para os pesquisadores interessados nesse campo.

Objetivo: Elaborar a versão em português do Psychotherapy Process Q-Set.

Método: A elaboração da versão em português do Psychotherapy Process Q-Set envolveu quatro etapas: tradução, retrotradução, avaliação da equivalência semântica e discussão, entre os autores, dos resultados. Para a aplicação do instrumento, cinco avaliadores foram treinados. Durante o treinamento, registros no diário de campo eram feitos para identificar dificuldades na execução da tarefa e subsidiar dados complementares. Após, o Psychotherapy Process Q-Set foi aplicado em sete sessões de uma psicoterapia psicodinâmica breve para examinar a concordância entre os juízes.

Resultados: A versão em português do Psychotherapy Process Q-Set apresentou boa equivalência semântica com a original. A avaliação da fidedignidade interavaliadores teve resultado satisfatório. Ressalta-se que a aplicação do Psychotherapy Process Q-Set requer estudo, tempo e reflexão. A discussão com os avaliadores apontou a necessidade de uma revisão do manual de aplicação no que diz respeito às vinhetas ilustrativas. Isto deverá ser realizado, futuramente, para minimizar as discrepâncias observadas no entendimento de alguns conceitos e para melhor adequá-las à realidade brasileira.

Conclusão: O estudo disponibiliza a versão em português do Psychotherapy Process Q-Set, um instrumento versátil, que pode ser utilizado em diferentes contextos para descrever, quantitativamente e em termos clinicamente significativos, o processo terapêutico das diferentes psicoterapias.

Descritores: Tradução, equivalência semântica, instrumentos, fidedignidade, processo terapêutico, psicoterapia.

\begin{abstract}
Introduction: In Brazil, psychotherapy research is in its early development; there are no systematic studies of the therapeutic process, and there are few available measurement instruments for researchers interested in this field.

Objective: To develop a Portuguese version of the Psychotherapy Process Q-Set.

Method: The development of a Portuguese version of the Psychotherapy Process Q-Set involved four stages: translation, back translation, evaluation of semantic equivalence and discussion of the results by the authors. Five raters were trained to apply the instrument. During the training, a field diary was used to record difficulties identified in task execution and to subsidize complementary data. Thereafter, the Psychotherapy Process Q-Set was applied to seven sessions of a short-term psychodynamic psychotherapy to examine agreement between referees.
\end{abstract}


Results: The Portuguese version of the Psychotherapy Process Q-Set presented good semantic equivalence with the original. The assessment of interrater reliability had a satisfactory result. It is worth stressing that applying the Psychotherapy Process Q-Set requires study, time and reflection. The discussion with raters pointed to the need of reviewing the application manual concerning the clinical examples. This will be performed in the near future to minimize the discrepancies observed in the understanding of some concepts and to better adjust them to the Brazilian reality.

Conclusion: This study provides a Portuguese version of the Psychotherapy Process Q-Set, a versatile instrument that can be used in different contexts to quantitatively describe the therapeutic process of different psychotherapies in clinically significant terms.

Keywords: Translation, semantic equivalence, instruments, reliability, therapeutic process, psychotherapy.

\section{Introdução}

Existe considerável consenso de que o impulso à pesquisa em psicoterapia possui origem na crítica feita por Hans Eysenck ${ }^{1}$ de que faltavam evidências que comprovassem ser as psicoterapias mais efetivas do que outros métodos de intervenção. A repercussão desse artigo foi quase imediata, gerando um grande número de estudos sobre a eficácia das psicoterapias e da psicanálise. Assim, durante mais de quatro décadas, os pesquisadores produziram consideráveis evidências sobre os resultados das psicoterapias ${ }^{2}$, de modo que, atualmente, a questão genérica de se as psicoterapias são ou não eficazes deixou de ser a preocupação dominante ${ }^{3,4}$. Desde a última década, novos problemas têm ocupado os pesquisadores, tais como: quais as psicoterapias que funcionam melhor para quais pacientes ${ }^{5} \mathrm{E}$ quais os aspectos, métodos e fatores que contribuem para a mudança em psicoterapia? ${ }^{6}$

Russell \& Orlinsky ${ }^{7}$ dividem a história da pesquisa em psicoterapia em quatro etapas, sendo que a atual apresenta um crescente interesse pelo estudo do processo terapêutico. Referindo-se especificamente à pesquisa psicanalítica, Wallerstein ${ }^{8}$ descreve quatro gerações sucessivas. A primeira teve início com o levantamento de Coriat sobre resultados terapêuticos, em 1917, e caracteriza-se por estudos de levantamento, baseado em "opiniões” e sem controle de vieses. A segunda teve início no final da década de 60, na Europa e EUA: são estudos em maior escala, prospectivos e comparativos, com múltiplas medidas e mais rigor no tratamento das variáveis. A terceira geração, contemporânea à segunda, alia a avaliação do processo com os resultados terapêuticos, mediante a aplicação de múltiplas medidas através do tempo. A quarta geração encontra-se in statu nascendi e concentra seu questionamento na natureza do processo terapêutico, investigado em profundidade através de gravações em áudio e vídeo.

O estudo microscópico da interação terapêutica, em sessões completas ou em fragmentos de sessões gravadas e transcritas, é ainda "música para o futuro", como pode ser observado na revisão sobre pesquisa psicanalítica levada a cabo por Fonagy et al. ${ }^{9}$, na qual, dos 50 projetos listados, apenas nove eram de estudos "puros" de processo e seis de processo/resultado. Na realidade, muitos estudos ditos de processo são, de fato, microestudos de resultados ${ }^{4}$.

O objetivo fundamental dos estudos sobre processo terapêutico é entender como ocorre a mudança ao longo do curso do tratamento ${ }^{2}$, isto é, identificar os mecanismos de ação terapêutica. Para tanto, os pesquisadores aplicam métodos qualitativos e quantitativos para examinar os padrões de interação e da comunicação terapeuta/paciente através das sessões de psicoterapia, relacionando-os com episódios terapêuticos positivos e negativos, bem como com a mudança clínica ${ }^{10}$.

Desde os primórdios, a psicanálise se ocupou do estudo do processo terapêutico do ponto de vista clínico. Não é por acaso, portanto, que muitos dos instrumentos criados nas últimas décadas para a investigação sistemática do processo terapêutico tenham sido desenvolvidos por pesquisadores dessa orientação, entre eles: o Fundamental Repetitive and Maladaptative Emotion Structures (FRAMES) ${ }^{11}$, o Core Conflictual Relationship Theme Method (CCRT) ${ }^{12}$ e o Psychotherapy Process Q-Set (PQS) ${ }^{13}$, alvo do presente estudo.

O PQS, assim como os demais instrumentos citados, baseia-se na transcrição literal das sessões de tratamento gravadas, questão que há várias décadas suscita controvérsias entre clínicos e pesquisadores psicanalíticos ${ }^{9,14-16}$.

No Brasil, a pesquisa em psicoterapia encontrase em estado inicial, e ainda não há estudos sistemáticos, em profundidade, do processo terapêutico. Os debates sobre o uso do gravador praticamente inexistem, salvo raras exceções ${ }^{17,18}$. Também são poucos os pesquisadores que investigam os efeitos de variáveis do processo sobre os resultados de tratamento. Destacam-se, nesse sentido, os trabalhos do Núcleo de Estudos e Pesquisa em Psicoterapia Breve com a Escala Diagnóstica Adaptativa Operacionalizada ${ }^{19}$ e a pesquisa de Marcolino ${ }^{20}$ sobre o impacto da aliança terapêutica na psicoterapia breve. No Rio Grande do Sul, não há estudos empíricos quantitativos que envolvam a investigação da dimensão processo/resultados 
terapêuticos. Não obstante, alguns trabalhos recentes, sobre fatores do paciente associados à capacidade de formar aliança terapêutica ${ }^{21}$ e sobre a associação entre aliança terapêutica e transferência ${ }^{22}$, denotam o interesse pela temática. Observa-se, ainda, um maior interesse, entre pesquisadores, na elaboração de versões brasileiras de instrumentos que avaliam fatores (do paciente ou da dupla paciente/terapeuta) que integram ou influenciam o processo da psicoterapia, tais como transferência ${ }^{22,23}$, contratransferência ${ }^{24}$, aliança terapêutica ${ }^{25}$ e mecanismos de defesa ${ }^{26}$. Tais iniciativas são fundamentais para o desenvolvimento, o aperfeiçoamento e a expansão da pesquisa em psicoterapia em nosso meio.

O estudo minucioso do processo terapêutico possibilita a geração e a avaliação de modelos explicativos de ação terapêutica ${ }^{13}$. Permite também examinar a validade da hipótese, ou veredicto Dodô ${ }^{27}$, que afirma que as diferentes psicoterapias são igualmente efetivas*. Embora corroborada por diversas pesquisas, essa hipótese tem sido questionada por alguns pesquisadores, que consideram a equivalência das psicoterapias um mito que reflete as limitações das variáveis em estudo ${ }^{28}$ e dos delineamentos e procedimentos de investigação utilizados ${ }^{29,30}$. Na realidade, parece razoável que diferentes técnicas produzam resultados semelhantes quando não se examina os meios através dos quais se produziram os resultados ${ }^{4}$. Portanto, a noção de que fatores nãoespecíficos (por exemplo, da aliança terapêutica) são elementos-chave para explicar os resultados terapêuticos deve-se, em parte, à falta de estudos sistemáticos, que investiguem os diferentes efeitos de diferentes tratamentos ou que identifiquem a relação entre as diferentes intervenções terapêuticas e os resultados ${ }^{30}$.

De modo geral, considera-se que fatores técnicos (específicos) diferenciam determinados tipos de tratamento, enquanto que fatores comuns (inespecíficos), tais como a relação entre terapeuta e paciente, estão sempre presentes. De fato, diversas pesquisas têm demonstrado que a aliança terapêutica é um importante preditor de resultados terapêuticos em diferentes psicoterapias ${ }^{31-33}$. Não há ainda, no entanto, estudos suficientes para esclarecer como tais fatores,

\footnotetext{
* A alusão ao veredicto do pássaro Dodô (“todo mundo ganhou e todos devem ganhar prêmios”) ao término da corrida, em comitê, no clássico Alice no país das Maravilhas, de Lewis Carroll, foi originalmente feita por Rosenzweig, em 1936, em um artigo no qual o autor introduz o conceito de fatores comuns nas psicoterapias. A utilização posterior, por Luborsky et al., em 1975, tornou-se consagrada na literatura e faz referência aos resultados dos numerosos estudos comparativos que falham em demonstrar diferenças significativas nos resultados de diferentes psicoterapias.
}

específicos ou comuns, contribuem para a mudança em psicoterapia $^{34}$.

Com o objetivo de compreender o papel dos diferentes fatores envolvidos no processo terapêutico é que foi desenvolvido o $\mathrm{PQS}^{\dagger}$. Esse instrumento, aplicável à sessão terapêutica gravada em áudio e/ou vídeo (unidade de análise), promove uma descrição detalhada e abrangente dos elementos do processo terapêutico em termos clinicamente relevantes e, ao mesmo tempo, compatíveis com a análise quantitativa e comparativa, contribuindo, assim, para a superação da histórica dissociação entre a atividade clínica e a pesquisa em psicoterapia ${ }^{13}$. O PQS tem sido utilizado, com sucesso, para estabelecer, empiricamente, relações causais entre o processo terapêutico e a mudança psíquica em delineamentos de caso único ou de múltiplos $\operatorname{casos}^{9}$. Foi baseado nesses estudos que Jones ${ }^{13,35}$ formulou o construto "estruturas de interação” (aspectos manifestos, comportamentais e emocionais da transferência e contratransferência) e desenvolveu uma teoria de ação terapêutica (da psicanálise e psicoterapia psicanalítica) que conjuga os efeitos do insight e do relacionamento paciente/ terapeuta sobre os resultados do tratamento. Além dos estudos causais, o PQS tem sido utilizado para comparar o processo terapêutico de diferentes psicoterapias $^{34,36,37}$, identificar os fatores do processo que melhor predizem os resultados terapêuticos ${ }^{30,38}$, investigar diferenças entre fases de tratamento ${ }^{39,40}$, examinar o processo de formulação das hipóteses clínicas dos psicoterapeutas ${ }^{41}$, determinar protótipos de diferentes tratamentos psicanalíticos e psicoterápicos ${ }^{42,43}$, entre outras aplicações descritas na literatura.

O presente artigo apresenta as etapas de elaboração da versão em português do PQS e descreve o estudo preliminar realizado para verificar a fidedignidade entre diferentes avaliadores, após o treinamento dos mesmos. É parte integrante de um projeto mais amplo, denominado "Relação entre processo e resultado em psicoterapia psicodinâmica breve: um estudo de caso”, uma investigação intensiva e sistemática de caso único, que envolve a aplicação do PQS nas 32 sessões de psicoterapia de uma paciente deprimida, cujo objetivo principal é compreender a interação entre as múltiplas variáveis do processo e o desfecho do tratamento.

\footnotetext{
† O instrumento é de autoria do psicólogo, psicanalista, professor e pesquisador da Universidade de Berkeley Enrico E. Jones, falecido em 2003. O PQS e seu manual original são de 1985 . Foram publicados pela primeira vez em 2000, no apêndice do livro Therapeutic action: $a$ guide to psychoanalytic therapy.
} 


\section{Método}

\section{Instrumento}

O PQS é um instrumento baseado na metodologia Q, também denominada método de ordenamento $\mathrm{Q}$ ou simplesmente método Q. Criado em 1935 pelo físico e psicólogo William Stephenson para estudar a subjetividade na perspectiva da própria pessoa ${ }^{44}, 0$ método foi posteriormente adaptado e modificado por Block $^{45}$ para permitir avaliações através de juízes externos. Em essência, seu propósito é fornecer uma descrição de um evento através do ordenamento $(Q$ Sort) de uma série de itens que descrevem uma opinião, característica, aspecto psicológico ou comportamental de um indivíduo ou situação. Uma particularidade do método é que não existe um conjunto Q (Q-Set) padrão; seu objetivo é justamente prover um conjunto de elementos que descrevam da melhor maneira as dimensões de variação do fenômeno em estudo ${ }^{45}$. Outra característica central do método é que os itens são avaliados em relação uns com os outros e não isoladamente ${ }^{45,46}$. Isso geralmente é realizado com o auxílio de cartões que contêm impressos os itens do instrumento, de modo que os mesmos possam ser ordenados em pilhas horizontais (categorias do um continuum prédefinido) em uma mesa de trabalho ${ }^{46}$.

O PQS possui 100 itens apresentados em cartões individuais e um manual explicativo com descrições e exemplos operacionais dos itens, para minimizar possíveis variações na interpretação dos mesmos ${ }^{13}$. Os itens podem ser classificados em três grandes grupos: 1) atitudes, comportamentos ou experiências do paciente; 2) ações e atitudes do terapeuta; 3) interação paciente/terapeuta ou clima terapêutico ${ }^{30}$.

A utilização do PQS requer treinamento e um estudo cuidadoso do manual. O procedimento de aplicação pode ser assim resumido: após o exame do material da sessão terapêutica e formulação inicial sobre os dados, os avaliadores (juízes) devem distribuir os cartões em nove pilhas, variando num continuum que vai do menos característico (categoria 1) ao mais característico (categoria 9). O número de cartões em cada pilha é distribuído em conformidade com a curva normal, variando de cinco cartões nos extremos a 18 cartões nas categorias do meio ${ }^{13}$. Essa distribuição forçada faz com que os avaliadores tenham que buscar o melhor arranjo para descrever os fenômenos, levando em consideração a freqüência, a intensidade e a importância de um item em relação aos demais, o que requer tempo e reflexão.

Os itens do PQS foram construídos a partir dos itens de outras medidas de processo terapêutico existentes e itens construídos por experts. Muitas versões foram testadas em estudos-piloto realizados em horas de tratamentos psicanalíticos e psicoterapêuticos de variadas orientações. Os itens que mostraram pouca variação entre uma ampla gama de sujeitos e sessões terapêuticas, que eram redundantes ou que apresentaram baixa fidedignidade interavaliadores foram excluídos. Revisões nos itens também foram realizadas cada vez que alguma faceta do processo terapêutico considerada relevante não era capturada pelo instrumento. A versão final mostrou-se capaz de avaliar uma variedade de fenômenos próprios do processo psicanalítico, tais como transferência, resistência, intervenções do terapeuta e estados afetivos do paciente ${ }^{39}$, bem como de outras orientações teóricas ${ }^{30}$.

Diversos estudos demonstram que a versão original do PQS apresenta boa fidedignidade interavaliadores ${ }^{13,30,36}$, validade de construto ${ }^{30} \mathrm{e}$ validade discriminante ${ }^{36}$. A validade fatorial é irrelevante, pois a medida supõe independência entre os itens. De fato, as investigações envolvendo a análise fatorial do PQS revelaram ausência de estrutura fatorial, o que é bastante desejável do ponto de vista da metodologia $\mathrm{Q}^{30}$.

O PQS já foi traduzido para espanhol (Ávila Espada ADA, Epstein R, Roussos A, Vidal Didier J, Winkel R, Traducción al español del manual de PQS, Berkeley University of California, 2001), alemão $0^{40} \mathrm{e}$ italiano ${ }^{37}$. Nos estudos realizados com essas versões, foram encontrados índices satisfatórios de concordância entre os juízes ${ }^{37,40,41}$. Além disso, a pesquisa realizada por Roussos ${ }^{41}$ demonstrou ser 0 PQS um instrumento sensível e capaz de discriminar hipóteses clínicas de psicoterapeutas cognitivos e psicanalíticos em segmentos de uma mesma sessão, um achado importante, visto que o PQS foi originalmente construído para a análise das sessões terapêuticas como um todo e não de suas partes.

Não existe um levantamento padrão dos resultados obtidos com o PQS, pois este pode variar conforme os objetivos da investigação em curso. Tipicamente, utiliza-se os itens pontuados nos extremos do ordenamento Q (mais e menos característicos) e/ou os agrupamentos obtidos mediante procedimentos de análise fatorial para se obter descrições sumarizadas do processo terapêutico (narrativas clínicas), que podem ser utilizadas em análises estatísticas inferenciais, quando necessário.

\section{Procedimentos}

A autorização para desenvolver e utilizar a versão em português do PQS foi obtida com o autor, 
Dr. Enrico Jones, através de comunicação eletrônica, em agosto de 2002.

A versão em português foi elaborada em quatro etapas. Na primeira, duas traduções independentes do manual e dos itens do instrumento foram realizadas por duas psicólogas bilíngües: uma das autoras deste artigo (F.B.S.) e outra, com vasta experiência em tradução, que não possuía conhecimento prévio do instrumento. A seguir, com o auxílio de um psiquiatra, também bilíngüe, foi desenvolvida a versão consensual, levando em consideração as duas versões preliminares. Em alguns itens, optou-se por uma ou outra versão e, em outros, por uma conjugação de ambas.

A segunda etapa foi a retrotradução da versão consensual para o idioma do original, o inglês. Essa etapa foi realizada por uma tradutora bilíngüe, experiente em tradução e retrotradução de instrumentos de pesquisa cuja língua nativa é o português.

A terceira etapa foi a avaliação da equivalência semântica feita por outros dois autores (M.L.T.N. e C.L.E.), tendo por base o modelo proposto por Herdman $^{47}$, já utilizado no Brasil por outros pesquisadores ${ }^{48-50}$. A avaliação levou em consideração os significados referencial e geral. A avaliação do significado referencial compreende a avaliação da medida em que as palavras da retrotradução referem as mesmas idéias ou objetos do original, isto é, a correspondência literal entre elas. Para cada item, foi utilizada uma escala analógica visual que permite a pontuação de 0 a $100 \%$. Já a avaliação do significado geral leva em consideração, além da equivalência literal, aspectos mais sutis da equivalência entre as versões (original e versão 1), tais como a manutenção do significado e o impacto que determinadas palavras ou expressões possuem no contexto cultural brasileiro. Os itens foram classificados em quatro categorias: inalterado, pouco alterado, muito alterado e completamente alterado.

A etapa seguinte foi a discussão, entre todos os autores, do resultado das avaliações e a modificação de alguns dos itens para atender aos critérios de equivalência semântica mencionados. Essa versão, juntamente com o manual, foi revisada por uma especialista em língua portuguesa para pequenos ajustes na redação e concordância verbal, resultando na versão final (vide Anexo 1).

Após a elaboração final da versão em português do PQS e do seu manual de codificação, realizou-se um treinamento com os avaliadores que irão atuar como juízes no estudo de caso já mencionado. Os avaliadores são cinco psicoterapeutas com experiência clínica variável (entre 3 e 14 anos), sendo uma médica especialista em psiquiatria e quatro psicólogas, das quais três possuem treinamento formal em psicoterapia psicanalítica em uma instituição local, cujo modelo de formação baseiase no "tripé": seminários teóricos, supervisão e tratamento pessoal.

$\mathrm{O}$ treinamento dos avaliadores foi coordenado por uma das autoras (F.B.S.) e realizado em aproximadamente 30 horas, distribuídas em nove encontros. Inicialmente, houve um intenso e cuidadoso estudo do manual de aplicação do PQS. Após, as transcrições de quatro sessões de uma psicoterapia psicodinâmica breve, que não do caso principal em estudo, foram examinadas, uma a uma, por todo o grupo. O ordenamento dos itens do PQS, nessas sessões, foi realizado através de consenso. Durante o treinamento, anotações em diário de campo eram feitas para identificar as eventuais dificuldades enfrentadas pelos avaliadores na execução da tarefa e para subsidiar dados complementares.

Finalmente, sete sessões de um terceiro caso de psicoterapia psicodinâmica breve foram distribuídas entre os avaliadores recém-treinados, sorteados em duplas, para que realizassem o ordenamento dos 100 itens do PQS, de modo independente. Usando o coeficiente de correlação de Pearson, foi então avaliada a concordância entre as duplas de avaliadores, tendo como parâmetro de fidedignidade interavaliadores coeficientes maiores ou iguais a 0,50 , critério adotado em outros trabalhos com o

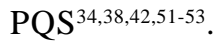

\section{Resultados}

De modo geral, os itens obtiveram boa equivalência entre as versões. Dos 100 itens do instrumento, 88 apresentaram significado referencial acima de $80 \%$. Com relação ao significado geral, 91 itens foram avaliados como possuindo significado idêntico (inalterado), oito, um pouco alterado, e um, com significado completamente alterado.

A Tabela 1 apresenta, a título de exemplo, alguns dos itens que geraram mais discussão, quer por apresentar significado referencial baixo, quer por apresentar significado geral alterado. 
Tabela 1 - Exemplo da análise da equivalência semântica dos itens do PQS

\begin{tabular}{|c|c|c|c|c|c|}
\hline Versão original (ING) & Versão consensual & Retrotradução (ING) & SR & SG & Versão final \\
\hline $\begin{array}{l}\text { Item 19: There is an } \\
\text { erotic quality to the } \\
\text { therapy relationship. }\end{array}$ & $\begin{array}{l}\text { em 19: Existe um tom } \\
\text { ótico na relação } \\
\text { rapêutica. }\end{array}$ & $\begin{array}{l}\text { Item 19: There is some } \\
\text { eroticism in the therapeutic } \\
\text { relationship. }\end{array}$ & $70 \%$ & $\mathrm{IN}$ & $\begin{array}{l}\text { xiste um tom } \\
\text { relação } \\
\text { a. }\end{array}$ \\
\hline $\begin{array}{l}\text { Item 34: Patient blames } \\
\text { others, or external } \\
\text { forces, for difficulties. }\end{array}$ & $\begin{array}{l}\text { m 34: O paciente culpa } \\
\text { ros, ou forças externas, } \\
\text { as suas dificuldades. }\end{array}$ & $\begin{array}{l}\text { Item 34: The patient blames } \\
\text { others, or external forces, for } \\
\text { his own difficulties. }\end{array}$ & $50 \%$ & $\mathrm{CA}$ & as, \\
\hline $\begin{array}{l}\text { Item 49: The patient } \\
\text { experiences ambivalent } \\
\text { or conflicted feelings } \\
\text { about the therapist. }\end{array}$ & $\begin{array}{l}\text { Item 49: O paciente tem } \\
\text { sentimentos ambivalentes } \\
\text { ou contrários sobre o } \\
\text { terapeuta. }\end{array}$ & $\begin{array}{l}\text { Item 49: The patient } \\
\text { experiments ambivalent or } \\
\text { antagonic feelings towards } \\
\text { the therapist. }\end{array}$ & $80 \%$ & $\mathrm{PA}$ & $\begin{array}{l}\text { Item 49: O paciente tem } \\
\text { sentimentos ambivalentes } \\
\text { ou conflituados sobre o } \\
\text { terapeuta. }\end{array}$ \\
\hline $\begin{array}{l}\text { Item 62: Therapist } \\
\text { identifies a recurrent } \\
\text { theme in the patient's } \\
\text { experience or conduct. }\end{array}$ & $\begin{array}{l}\text { Item 62: O terapeuta } \\
\text { identifica um tema } \\
\text { recorrente na experiência } \\
\text { ou conduta do paciente. }\end{array}$ & $\begin{array}{l}\text { Item 62: The therapist } \\
\text { identifies a recurrent subject } \\
\text { in the experience or conduct } \\
\text { of the patient. }\end{array}$ & $90 \%$ & PA & $\begin{array}{l}\text { Item 62: O terapeuta } \\
\text { identifica um tema } \\
\text { repetitivo na experiência } \\
\text { ou conduta do paciente. }\end{array}$ \\
\hline
\end{tabular}

CA = completamente alterado; IN = inalterado; ING = inglês; PA = parcialmente alterado; $\mathrm{SG}$ = significado geral; $\mathrm{SR}=$ significado referencial.

Durante o treinamento com o PQS, foram identificadas algumas falhas na compreensão e no manejo inicial do instrumento. Entre os problemas de compreensão, destacou-se a pouca familiaridade dos avaliadores em treinamento com alguns conceitos oriundos de outras abordagens psicoterápicas que não a psicanalítica (por exemplo, na definição do item 80, "o terapeuta apresenta uma experiência ou evento numa perspectiva diferente”, é referido o conceito “reestruturação cognitiva”). Nas discussões realizadas entre os participantes do treinamento, foi assinalado que seria desejável que o manual do PQS apresentasse vinhetas clínicas ilustrativas em todos os itens e não apenas em alguns. Uma das vinhetas foi considerada pouco adequada à nossa realidade, embora não estivesse causando prejuízo no entendimento do item. Trata-se do exemplo que acompanha o item 73 (“o paciente está comprometido com o trabalho terapêutico”): “um paciente está tão interessado em começar o tratamento que está disposto a abrir mão de seu jogo de golfe semanal para poder manter as consultas da terapia”. Outra observação importante foi que o grupo de avaliadores, especialmente nos primeiros encontros de treinamento, apresentou dificuldade em assumir a postura de um observador neutro requerida nas instruções do instrumento ${ }^{13}$. Também foi detectada, nessa etapa, a tendência a interpretar o material da sessão, ao invés de simplesmente descrevê-lo.

Findo o treinamento, foi realizado o estudo de fidedignidade interavaliadores. Das sete sessões avaliadas, em cinco foi encontrada boa fidedignidade (coeficientes de correlação de Pearson entre 0,53 e 0,64) com apenas dois juízes. Nas outras duas sessões, foi necessária a inclusão de um terceiro avaliador para produzir índices semelhantes $(r=0,60$ e $r=0,72)$.

\section{Discussão}

Com relação ao estudo de equivalência semântica do PQS, considerando as duas avaliações realizadas, percebe-se que a do significado referencial é a que se mostrou com mais problemas. É digno de nota que vários itens que tiveram avaliação do significado referencial baixa foram avaliados como apresentando significado geral inalterado, o que indica a existência de algumas dificuldades na retrotradução em itens que a tradução mostrou-se adequada. A explicação para isso pode ser encontrada no fato de a retrotradução ter sido realizada por profissional não familiarizado com a linguagem do instrumento, especialmente palavras e expressões que representam jargão da técnica em psicoterapia, e também por se tratar de pessoa cuja língua nativa é o português e não o inglês. Tendo isso em consideração, foram feitos os ajustes necessários na tradução. E, após a revisão final, considerou-se que ambas as versões, a original e a traduzida para o português, eram equivalentes do ponto de vista semântico.

Uma vez que o PQS é um instrumento cuja aplicação depende de um treinamento especial dos juízes, recomenda-se a avaliação da fidedignidade interavaliadores sempre que for utilizado. Para nós, foram necessárias 30 horas de treinamento, distribuídas em encontros quinzenais ao longo de 5 meses, sendo que, nos intervalos entre os encontros, os avaliadores 
estudavam o material clínico e realizavam suas avaliações individuais para, então, discutir no grupo.

Consideramos o resultado do estudo de fidedignidade animador, visto que a avaliação do processo terapêutico através do PQS é extremamente detalhada, requerendo paciência e cuidado dos avaliadores ${ }^{13}$, além de, obviamente, uma boa dose de intuição e julgamento clínico.

É digno de nota que o grupo de avaliadores tinha experiência clínica variável e que não se observou qualquer tendência no sentido da influência desta sobre as avaliações, quer durante o treinamento, quer durante o estudo recém-mencionado. As dificuldades identificadas nesses momentos dizem respeito, sobretudo, à maior ou menor complexidade dos fenômenos presentes nas sessões, embora também tenham ocorrido algumas falhas na compreensão exata de alguns itens em avaliação por parte dos avaliadores. Quanto à primeira dificuldade identificada, nada há a fazer além de reconhecê-la e tentar dedicar mais tempo ao estudo das "sessões difíceis", ou seja, sessões onde os estados mentais e atitudes do paciente e/ou do terapeuta não são facilmente depreendidos do material escrito. Isso pode ocorrer, por exemplo, quando a interação não-verbal predomina ou quando o conteúdo do discurso é vago, difuso ou caótico.

Já para melhorar a compreensão dos itens, fizemos um levantamento daqueles cuja avaliação entre juízes foi mais discrepante e novamente reunimos o grupo de avaliadores para discuti-los em conjunto. Essa parece ser uma dificuldade facilmente contornável através de mais treinamento. Por outro lado, uma vez que, nesse grupo de avaliadores, os exemplos clínicos foram considerados essenciais para a plena compreensão dos itens, planejamos uma adaptação do manual com a inclusão de novas vinhetas ilustrativas, levando em consideração a nossa realidade. A adaptação do manual às necessidades de determinado projeto de pesquisa, a exemplo do que outros pesquisadores já fizeram ${ }^{37}$, é um recurso que o pesquisador possui para aumentar a confiabilidade do instrumento e minimizar as divergências entre as avaliações dos diferentes juízes.

Com relação às dificuldades iniciais dos avaliadores em manter um distanciamento ótimo do material examinado e em descrever os eventos ao invés de interpretá-los, os autores da versão em português do PQS acreditam ser perfeitamente compreensível e até esperado que isso ocorra, visto que o grupo é formado por psicoterapeutas clínicos e não por pesquisadores. Assim, todos os participantes possuíam experiência prévia com relatos (de memória) de sessões de psicoterapia para fins de supervisão, situação onde a interpretação assume maior relevância que a descrição. Além disso, nenhum dos avaliadores tinha tido antes a oportunidade de examinar um relato de sessão audiogravada, no qual as particularidades do discurso do paciente e do terapeuta (por exemplo, expressões verbais, silêncios e lapsos de linguagem) são captadas com maior precisão.

Como é sabido, nos relatos de memória, o texto (por exemplo, as intervenções do terapeuta) está “lapidado" pela ação do processo secundário. O material clínico usualmente levado à supervisão é uma versão revisada, corrigida e censurada dos eventos do tratamento ${ }^{54}$. Já nos relatos de gravação em áudio ou vídeo essa modificação está ausente, o que pode causar maior impacto emocional no leitor.

Especialmente no início do treinamento, os avaliadores tendiam a fazer comentários sobre a adequação das intervenções do terapeuta do caso (questão não avaliada pelo PQS). Essa falha na neutralidade, registrada no diário de campo, está de acordo com a afirmação de Sandler \& Sandler ${ }^{55}$ de que o material clínico proveniente de gravações freqüentemente causa a impressão, nos demais colegas, de que o analista (ou terapeuta, nesse caso) é um mau analista.

É importante destacar que, na medida em que os avaliadores foram se familiarizando com o tipo de material clínico e com os procedimentos de aplicação do PQS, uma maior objetividade na execução da tarefa foi atingida. Isso pôde ser verificado tanto nos relatos dos participantes como no resultado do exame da concordância entre avaliadores.

\section{Conclusão}

O estudo disponibiliza, aos interessados na pesquisa em psicoterapia no Brasil, a versão em português do PQS, um instrumento versátil e capaz de fornecer descrições empírica e clinicamente significativas do processo terapêutico de diferentes abordagens psicoterápicas. Tais descrições, compatíveis com a análise quantitativa, têm sido utilizadas há duas décadas por diversos pesquisadores, em variados contextos, no estudo do processo terapêutico em delineamentos grupais e em estudos de caso. A aplicação do PQS requer treinamento, tempo e reflexão. O estudo preliminar realizado com a versão em português do instrumento demonstrou significativo consenso entre os julgamentos clínicos dos diferentes avaliadores. O extenso treinamento realizado previamente com os avaliadores demonstrou ser esse procedimento essencial para a compreensão e manejo adequado do PQS. Uma revisão do manual, no que diz respeito às vinhetas clínicas ilustrativas, poderá minimizar as dificuldades encontradas na compreensão plena de alguns dos itens que refletem conceitos pouco familiares aos avaliadores. 


\section{Agradecimentos}

A Karina Brodski e Luís Guilherme Streb, pelo auxílio na tradução do PQS e do seu manual de aplicação; a Miriam Simões Pires, pelo trabalho de retrotradução dos itens do PQS; a Maria de Lourdes Figueiredo Leal, pela revisão do português realizada nos itens e manual do PQS; a Aline Eymael Domingues, Andréia Chaieb, Karen Selister, Patrícia Aronis e Roberta Rossi Grüdtner, pela participação no treinamento, pelo trabalho de aplicação do PQS e pelas sugestões para a adaptação futura do seu manual de aplicação; aos psicoterapeutas e pacientes anônimos que consentiram ter seus tratamentos gravados para a execução de nosso estudo.

\section{Referências}

1. Eysenck HJ. The effects of psychotherapy: an evaluation. J Consult Psychol. 1952;16(5):319-24.

2. Drozd JF, Goldfried MR. A critical evaluation of the state-of-the-art in psychotherapy outcome research. Psychotherapy 1996;33(2):17180.

3. Lambert MJ. Introduction to psychotherapy research. In: Beutler LE, Crago MM, eds. Psychotherapy research: an international review of programmatic studies. Washington: Am Psychol Assoc; 1991. p. 1-23.

4. Poch J, Ávila Espada A. Investigación en psicoterapia: la contribución psicoanalítica. Barcelona: Paidós; 1998.

5. Roth A, Fonagy P. What works for whom? A critical review of psychotherapy research. 2nd. ed. New York: Guilford; 2005.

6. Whiston SC, Sexton TL. An overview of psychotherapy outcome research: implications for practice. Prof Psychol Res Pr. 1993;24(1):43-51.

7. Russell RL, Orlinsky DE. Psychotherapy research in historical perspective. Arch Gen Psychiatry. 1996;53(8):708-15.

8. Wallerstein R. The generations of psychotherapy research: an overview. In: Leuzinger-Bohleber $\mathbf{M}$, Target $\mathbf{M}$, eds. Outcomes of psychoanalytic treatment: perspectives for therapists and researchers. London: Whure; 2002. p. 30-52.

9. Fonagy P. An open door review of outcome studies in psychoanalysis. London: International Psychoanalytic Association: 1999.

10. Russell R. Introduction to special section on multivariate psychotherapy process research: Structure and change in the talking cure. J Consult Clin Psychol. 1995;63(1):3-5.

11. Dahl H, Teller V. The characteristics, identification and application of FRAMES. Psychother Res. 1994;4:253-76.

12. Luborsky L, Popp C, Luborsky E, Mark D. The core conflictual relationship theme. Psychother Res. 1994;4:172-83.

13. Jones EE. Therapeutic action: a guide to psychoanalytic therapy. Northvale: Aronson; 2000.

14. Engel G. Some obstacles to the development of research in psychoanalysis. J Am Psychoanal Assoc. 1968;16(2):195-229.

15. Gill MM, Simon J, Fink G, Endicott NA, Paul IH. Studies in audiorecorded psychoanalysis. I. General considerations. J Am Psychoanal Assoc. 1968;16(2):230-44.

16. Kächele H, Thomä H, Ruberg W, Grünzig HJ. Audio recording of the psychoanalytic dialogue: scientific, clinical and ethical problems. In: Dahl H, Kächele H, Thomä H, eds. Psychoanalytic process research strategies. Berlin: Springer; 1988. p. 179-93.

17. Nunes MLT. O uso do gravador para pesquisa em psicoterapia. Rev Psico. 1995;26(2):121-32.

18. Domingues AE, Serralta, FB. O uso do gravador na psicoterapia psicanalítica: o ponto de vista do psicoterapeuta. Rev Bras Psicoter. 2005;7(2/3):169-82.

19. Yoshida EMP, Enéas MLE. A proposta do Núcleo de Estudos e
Pesquisa em Psicoterapia para adultos. In: Yoshida EMP, Enéas MLE, orgs. Psicoterapias psicodinâmicas breves. Propostas atuais. Campinas: Alínea; 2004. p. 223-58.

20. Marcolino JAM. O impacto da aliança terapêutica em psicoterapia psicodinâmica breve [tese]. São Paulo: Universidade de São Paulo; 2002.

21. Gomes FG. A relação entre os mecanismos de defesa e a qualidade da aliança terapêutica em psicoterapia de orientação analítica de adultos: um estudo exploratório [dissertação]. Porto Alegre: Universidade Federal do Rio Grande do Sul; 2003.

22. Ferreira EB. Um estudo sobre o padrão transferencial e a aliança terapêutica de pacientes em psicoterapia de orientação analítica [dissertação]. Porto Alegre: Universidade Federal do Rio Grande do Sul; 2006.

23. Bottino SMB. Estudo da sistematização do diagnóstico em psicoterapia através do CCRT: tema central de conflito nos relacionamentos [dissertação]. São Paulo: Universidade de São Paulo; 2000.

24. Goldfeld PRM. Um estudo da contratransferência em um grupo de psicoterapeutas de orientação psicanalítica frente a relatos de situações traumáticas [dissertação]. Porto Alegre: Universidade Federal do Rio Grande do Sul; 2005.

25. Marcolino JAM, Iacoponi E. Escala de aliança psicoterápica da Califórnia na versão do paciente. Rev Bras Psiquiatr. 2001;23(2):88-

26. Blaya C, Kipper L, Heldt E, Isolan L, Ceitlin LH, Bond M, et al. Brazilian-Portuguese version of the Defense Style Questionnaire (DSQ-40) for defense mechanisms measure: a preliminary study. Rev Bras Psiquiatr. 2004;26(4):255-8.

27. Luborsky L, Singer B, Luborsky L. Comparative studies of psychotherapies. It is true that "everywon has one and all must have prizes?” Arch Gen Psychiatry. 1975;32(8):995-1008.

28. Beutler LE. Have all won and must all have prizes? Revisiting Luborsky et al.'s verdict. J Consult Clin Psychol. 1991;59(2):22632.

29. Stiles WB, Shapiro DA, Elliott R. Are all psychotherapies equivalent? Am Psychol. 1986;41(2):165-80.

30. Jones EE, Cumming JD, Horowitz MJ. Another look at the nonspecific hypothesis of therapeutic effectiveness. J Consult Clin Psychol. 1988;56(1):48-55.

31. Horvath A, Symonds BD. Relations between working alliance and outcome in psychotherapy: a meta-analysis. J Couns Psychol. 1991;38(2):139-49.

32. Westerman MA, Foote JP, Winston A. Change in coordination across phases of psychotherapy and outcome: two mechanisms for the role played by patients' contribution to the alliance. J Consult Clin Psychol. 1995;63(4):672-5.

33. Barber JP, Connolly MB, Crits-Christoph P, Gladis L, Siqueland L. Alliance predicts patients' outcome beyond in-treatment change in symptoms. J Consult Clin Psychol. 2000;68(6):1027-32.

34. Ablon JS, Jones EE. Psychotherapy process in the National Institute of Mental Health Treatment Depression Collaborative Research Program. J Consult Clin Psychol. 1999;67(1):64-75.

35. Jones EE. Therapeutic action: a new theory. Am J Psychother. 2001;55(4):460-74.

36. Jones EE, Pulos SM. Comparing process in psychodynamic and cognitive-behavioral therapies. J Consult Clin Psychol. 1993;61(2):306-16.

37. Sirigatti S. Application of the Jones' Psychotherapy Process Q-Sort. Brief Strategic Systemic Ther Eur Rev. 2004;1:194-207.

38. Ablon JS, Levy RA, Katzenstein T. Beyond brand names of psychotherapy: identifying empirically supported change processes. Psychother Theor Res Pract Train. 2006;43(2):216-31.

39. Jones EE, Windholz M. The psychoanalytic case study: toward a method for systematic inquiry. J Am Psychoanal Assoc. 1990;38(4):985-1015.

40. Albani C, Blaser G, Jacobs U, Jones E, Thomä H, Kächele H. Amalia $X$ 's psychoanalytic psychotherapy in the light of Jones's Psychotherapy Process Q-Sort (2002). In: Leuzinguer-Bohleber M, Target M. Outcomes of psychoanalytic treatment: perspectives for therapists and researchers. London: Whurr; 2002. p. 294-302.

41. Roussos AJ. La inferencia clínica y la elaboración de hipótesis de 
trabajo de los psicoterapeutas: estudio empírico mediante el uso de técnicas de análisis de procesos terapéuticos [tese]. Buenos Aires: Universidad de Belgrano; 2001.

42. Ablon JS, Jones EE. How expert clinicians' prototypes of an ideal treatment correlate with outcome in psychodynamic and cognitivebehavioral therapy. Psychother Res. 1998;8(1):71-83.

43. Epstein R, Murillo M, Barletta L. Investigación empírica sobre la sesión de psicoanalisis y la sesión de psicoterapia psicoanalítica [poster presentation]. In: 43rd World Congress of the International Psychoanalytic Association; 2004 Mar 10-14; New Orleans, USA.

44. Brown SR. Q methodology and qualitative research. Qual Health Res. 1996;6(4):561-7.

45. Block J. The Q-sort method in personality assessment and psychiatric research. Springfield: Charles C. Thomas; 1961.

46. McKeown B, Thomas D. Q methodology. Newbury Park: Sage; 1988.

47. Herdman M, Fox-Rushby J, Badia X. A model equivalence in the cultural adaptation of HRQoL instruments: the universalist approach. Qual Life Res. 1998;7(4):323-35.

48. Reichenheim M, Moraes CL, Hasselmann MH. Equivalência semântica da versão em português do instrumento Abuse Assessment Screen para rastrear a violência contra a mulher grávida. Rev Saude Publica 2000;34(6):610-6.
49. Moraes CL, Hasselmann MH, Reichenheim M E. Adaptação transcultural para o português do instrumento Revised Conflict Tactics Scales (CTS2), utilizado para identificar a violência entre casais. Cadernos de Saude Publica 2002;18(1):163-76.

50. Berger W, Mendlowicz MV, Souza WF, Figueira I. Equivalência semântica da versão em português da Post-Traumatic Stress Disorder Checklist - Civilian Version (PCL-C) para rastreamento do transtorno do estresse pós-traumático. Rev Psiquiatr Rio Gd. Sul. 2004;26(2):167-75.

51. Jones EE, Parke LA, Pulos SM. How therapy is conducted in the private consulting room: a multidimensional description of brief psychodynamic treatments. Psychother Res. 1992;2(1):16-30.

52. Price P, Jones EE. Examining the alliance using the Psychotherapy Process Q-Set. Psychotherapy: Theory, Research, Practice, Training. 1998;35(3):392-404.

53. Pole N, Jones EE. The talking cure revisited: psychodynamic psychotherapy content analyses of a two-year psychodynamic psychotherapy. Psychother Res. 1998;8(2):171-89.

54. Lancelle G. El psicoanalisis y la investigación en escorzo desde una perspectiva clínica. Psicoanálisis APdeBA. 1997;19(1-2):119-55.

55. Sandler J, Sandler AM. The past unconscious, the present unconscious and interpretation of the transference. Psychoanal Inq. 1985;4:367-99. 
Anexo 1 - Itens do Psychotherapy Process Q-Set

Item 1: O paciente verbaliza sentimentos negativos (por exemplo, crítica, hostilidade) dirigidos ao terapeuta (versus faz comentários de aprovação ou admiração).

Item 2: O terapeuta chama a atenção para o comportamento não-verbal do paciente, como, por exemplo, postura corporal, gestos.

Item 3: As observações do terapeuta visam facilitar a fala do paciente.

Item 4: Os objetivos do paciente no tratamento são discutidos.

Item 5: O paciente tem dificuldade para compreender os comentários do terapeuta.

Item 6: O terapeuta é sensível aos sentimentos do paciente, afinado com o paciente; empático.

Item 7: O paciente está ansioso ou tenso (versus calmo e descontraído).

Item 8: O paciente está preocupado ou conflituado com sua dependência do terapeuta (versus confortável com a dependência ou querendo a dependência).

Item 9: O terapeuta é distante, indiferente (versus responsivo e efetivamente envolvido).

Item 10: O paciente busca maior intimidade com o terapeuta.

Item 11: Sentimentos e experiências sexuais são discutidos.

Item 12: Ocorrem silêncios durante a sessão.

Item 13: O paciente está animado ou excitado.

Item 14: O paciente não se sente entendido pelo terapeuta.

Item 15: O paciente não inicia assuntos; é passivo.

Item 16: Há discussão sobre funções corporais, sintomas físicos ou saúde.

Item 17: O terapeuta exerce ativamente controle sobre a interação com o paciente (por exemplo, estruturando e/ou introduzindo novos assuntos).

Item 18: O terapeuta transmite um sentido de aceitação não-crítica (Obs.: a colocação na direção do extremo não-característico indica desaprovação, falta de aceitação).

Item 19: Existe um tom erótico na relação terapêutica.

Item 20: O paciente é provocador, desafia os limites da relação terapêutica (Obs.: a colocação na direção do extremo nãocaracterístico indica que o paciente se comporta de maneira submissa).

Item 21: O terapeuta revela informações sobre si.

Item 22: O terapeuta focaliza os sentimentos de culpa do paciente.

Item 23: O diálogo tem um foco específico.

Item 24: Os conflitos emocionais do próprio terapeuta invadem a relação.

Item 25: O paciente tem dificuldade em começar a sessão.

Item 26: O paciente experimenta afetos incômodos ou penosos (dolorosos).

Item 27: O terapeuta dá orientações e conselhos explícitos (versus adia, mesmo quando é pressionado a fazê-lo).

Item 28: O terapeuta percebe acuradamente o processo terapêutico.

Item 29: O paciente fala sobre querer estar separado ou distante.

Item 30: A discussão se concentra em temas cognitivos, isto é, sobre idéias ou sistema de crenças.

Item 31: O terapeuta solicita mais informação ou elaboração.

Item 32: O paciente adquire uma nova compreensão ou insight.

Item 33: O paciente fala sobre sentimentos de estar próximo ou de estar precisando de alguém.

Item 34: O paciente culpa outros, ou forças externas, pelas dificuldades.

Item 35: A auto-imagem é um foco de discussão.

Item 36: O terapeuta assinala o uso de manobras defensivas pelo paciente; por exemplo, anulação, negação.

Item 37: O terapeuta se comporta como um professor (de maneira didática).

Item 38: Há discussão sobre atividades ou tarefas específicas para o paciente tentar fazer fora da sessão.

Item 39: Existe um tom competitivo na relação. 
Item 40: O terapeuta faz interpretações que fazem referência a pessoas reais da vida do paciente (Obs.: a colocação na direção do extremo não-característico indica que o terapeuta faz interpretações gerais ou impessoais).

Item 41: $\quad$ As aspirações ou ambições do paciente são tópicos de discussão.

Item 42: O paciente rejeita (versus aceita) os comentários e observações do terapeuta.

Item 43: O terapeuta sugere o significado do comportamento de outros.

Item 44: O paciente se sente cauteloso ou desconfiado (versus confiante e seguro).

Item 45: O terapeuta adota uma atitude de apoio.

Item 46: O terapeuta se comunica com o paciente com um estilo claro e coerente.

Item 47: Quando a interação com o paciente é difícil, o terapeuta tenta se adaptar, num esforço para melhorar a relação.

Item 48: O terapeuta estimula a independência de ação ou opinião do paciente.

Item 49: O paciente tem sentimentos ambivalentes ou conflituados sobre o terapeuta.

Item 50: O terapeuta chama a atenção para sentimentos considerados inaceitáveis pelo paciente (por exemplo, raiva, inveja ou excitação).

Item 51: O terapeuta é condescendente ou protetor para com o paciente.

Item 52: O paciente conta com o terapeuta para resolver seus problemas.

Item 53: O paciente está preocupado com o que o terapeuta pensa dele.

Item 54: O paciente é claro e organizado em sua expressão.

Item 55: O paciente transmite expectativas positivas sobre a terapia.

Item 56: O paciente discute experiências como se estivesse distante dos seus sentimentos (Obs.: avalie como neutro se o afeto e o envolvimento forem aparentes, mas modulados).

Item 57: O terapeuta explica as razões por trás de sua técnica ou abordagem ao tratamento.

Item 58: O paciente resiste em examinar pensamentos, reações ou motivações relacionados aos problemas.

Item 59: O paciente se sente inadequado ou inferior (versus eficiente e superior).

Item 60: O paciente tem uma experiência catártica (Obs.: avalie como não-característico se a expressão emocional não for acompanhada de uma sensação de alívio).

Item 61: O paciente se sente tímido ou envergonhado (versus à vontade e seguro).

Item 62: O terapeuta identifica um tema repetitivo na experiência ou conduta do paciente.

Item 63: Os relacionamentos interpessoais do paciente são um tema importante.

Item 64: O amor ou relacionamentos amorosos são um tópico de discussão.

Item 65: O terapeuta clarifica, reafirma ou reformula a comunicação do paciente.

Item 66: O terapeuta é diretamente encorajador (Obs.: coloque na direção do não-característico se o terapeuta tende a se abster de proporcionar apoio direto).

Item 67: O terapeuta interpreta desejos, sentimentos ou idéias, rejeitadas ou inconscientes.

Item 68: Significados reais versus fantasiados das experiências são ativamente diferenciados.

Item 69: A situação de vida atual ou recente do paciente é enfatizada na discussão.

Item 70: O paciente luta para controlar sentimentos ou impulsos.

Item 71: O paciente é auto-acusatório; expressa vergonha ou culpa.

Item 72: O paciente entende a natureza da terapia e o que é esperado.

Item 73: O paciente está comprometido com o trabalho terapêutico.

Item 74: O humor é utilizado.

Item 75: Interrupções ou pausas no tratamento ou o término da terapia são discutidos.

Item 76: O terapeuta sugere que o paciente aceite responsabilidade por seus problemas.

Item 77: O terapeuta não tem tato.

Item 78: O paciente busca aprovação, afeição ou simpatia do terapeuta.

Item 79: O terapeuta comenta as mudanças no humor ou no afeto do paciente.

Item 80: $\quad$ O terapeuta apresenta uma experiência ou evento numa perspectiva diferente. 
Item 81: O terapeuta enfatiza os sentimentos do paciente para ajudá-lo a experimentá-los mais profundamente.

Item 82: O comportamento do paciente durante a sessão é reformulado pelo terapeuta de uma maneira não explicitamente reconhecida anteriormente.

Item 83: O paciente é exigente.

Item 84: O paciente expressa sentimentos de raiva ou agressivos.

Item 85: O terapeuta encoraja o paciente a tentar novas formas de comportar-se com os outros.

Item 86: O terapeuta é seguro ou autoconfiante (versus inseguro ou defensivo).

Item 87: O paciente é controlador.

Item 88: O paciente traz temas e material significativos.

Item 89: O terapeuta age para fortalecer defesas.

Item 90: Os sonhos ou fantasias do paciente são discutidos.

Item 91: Lembranças ou reconstruções da infância são tópicos de discussão.

Item 92: Os sentimentos ou percepções do paciente são relacionados com situações ou comportamentos do passado.

Item 93: O terapeuta é neutro.

Item 94: O paciente sente-se triste ou deprimido (versus alegre ou animado).

Item 95: O paciente sente-se ajudado.

Item 96: Há discussão sobre horários ou honorários.

Item 97: O paciente é introspectivo, prontamente explora pensamentos e sentimentos íntimos.

Item 98: A relação terapêutica é um foco de discussão.

Item 99: O terapeuta questiona a visão do paciente (versus valida as percepções do paciente).

Item 100: O terapeuta faz conexões entre a relação terapêutica e outras relações. 